\title{
O USO DO ÁLCOOL E OUTRAS DROGAS COMO FATOR SOCIAL ENTRE OS ACADÊMICOS DO CURSO DE PSICOLOGIA
}

\author{
Ana Rita Cardoso MEDEIROS ${ }^{1}$ \\ Sara Cristina de OLIVEIRA ${ }^{2}$ \\ Kimberly Marie JONES3 \\ Leonardo Augusto Couto FINELLI ${ }^{4}$ \\ Wellington Danilo SOARES ${ }^{5}$
}

\begin{abstract}
${ }^{1}$ Graduada em Psicologia pelas Faculdades de Saúde Ibituruna - FASI, Montes Claros, MG. Email: anaritaarc@hotmail.com.

${ }^{2}$ Graduada em Psicologia pelas Faculdades de Saúde Ibituruna - FASI, Montes Claros, MG. Email:sarinhacristina84@live.com

${ }^{3}$ Doutora em Antropologia pela Universidade Pittsburgh/Estados Unidos. Gestora de pesquisa das Faculdades Integradas do Norte de Minas - FUNORTE, Montes Claros - Minas Gerais, Brasil. Email: kimberly.jones@ funorte.edu.br

${ }^{4}$ Doutor em Ciências da Educação pela Universidad Evangélica Del Paraguay. Professor do curso de Psicologia das Faculdades de Saúde Ibituruna - FASI, Montes Claros, MG. Email: Leonardo.finelli@ funorte.edu.br

${ }^{5}$ Doutor em Ciências da Saúde pela Universidade Estadual de Montes Claros - Unimontes. Professor do curso de Psicologia das Faculdades de Saúde Ibituruna - FASI, Montes Claros, MG. Email: wdansoa@ yahoo.com.br
\end{abstract}

Recebido em: 20/06/2016 - Aprovado em: 20/11/2017 - Disponibilizado em: 30/12/2017

\begin{abstract}
RESUMO
O início precoce da ingestão de álcool é preocupante, uma vez que, quanto mais cedo inicia-se o consumo, maiores são as chances de se estabelecer a dependência dessa substância. O objetivo foi identificar a prevalência do consumo de álcool em acadêmicos de Psicologia de uma instituição de ensino superior da cidade de Montes Claros - MG. Estudo descritivo, quantitativo e transversal, realizado entre os meses de abril e maio de 2016. População constituída por acadêmicos do curso de Psicologia da cidade de Montes Claros - MG. Utilizou-se o Teste de Identificação de Desordens Devido ao Álcool (AUDIT), o Inventário de Expectativas e Crenças Pessoais acerca do Álcool (IECPA), o Teste de Triagem do Envolvimento com Álcool, Cigarro e Outras Substâncias (ASSIST), a Escala de Satisfação com o Suporte Social (ESSS). Os dados permitiram concluir que da amostra pesquisada registrou baixa prevalência de dependência do álcool e outras drogas lícitas e ilícitas.
\end{abstract}

Palavras-chave: Consumo. Álcool.Mediador Social.Acadêmicos.

\begin{abstract}
Early initiation of alcohol intake is troubling since the sooner it begins the consumption, the higher the chances of establishing the dependence of this substance. The objective wasidentifying the prevalence of alcohol consumption in psychology academics from a higher education institution of the city of Montes Claros - MG. Descriptive, quantitative and transversal, carried out between the months of April and May 2016. Population constituted of academics from the course of Psychology of the city of Montes Claros, Minas Gerais. Was used the Test of Disorders Identification Due to Alcohol (AUDIT), the Inventory of Expectations and Personal Beliefs about Alcohol (IECPA), the Involvement Screening Test with Alcohol, Cigarette and Other Substances (ASSIST), the Satisfaction Scale with Social Support (ESSS). With regarding the researched sample found a low prevalence with alcohol dependence and other licit and illicit drugs.
\end{abstract}

Keywords: Consumption.Alcohol.Social mediator.Academics. 


\section{INTRODUÇÃO}

De acordo com a definiçãa da Organização Mundial da Saúde (OMS 2011), droga é qualquer substância que introduzida no organismo atua sobre um ou mais de seus sistemas produzindo alterações em seu funcionamento (NICASTRI,2011).

Segundo Martins (2011), do ponto de vista legal, as drogas são classificadas como lícitas e ilícitas. Dizem-se lícitas as substâncias que possuem uso e comercialização permitidos pelo Estado, como a cafeína, o tabaco e o álcool. Em contraposição, as substâncias ilícitas correspondem àquelas que não possuem permissão do estado para comercialização e uso.

Considera-se uso nocivo, quando o consumo de substância psicoativa torna-se prejudicial à saúde, podendo trazer complicações físicas ou psíquicas. No que diz respeito à dependência, a OMS postula que essa síndrome ocorre através do uso persistente de uma substância psicoativa, que resulta em uma dificuldade de controle e desejo constante de ingerir a droga. A dependência traz prejuízos fisiológicos e compromete o comportamento e cognição do sujeito (MANGUEIRA, 2014).

O álcool é uma droga psicotrópica, cujo consumo é admitido e até mesmo incentivado pela sociedade, o que leva a uma diferenciação de seu uso, em comparação às demais drogas (CEBRID, 2013). Essa prerrogativa sustenta o exposto por Nicastri (2011), quando afirmam que a permissividade leva à falsa crença de inocência do seu uso, culminando no consumo excessivo, o que tem se tornado um dos principais problemas das sociedades modernas.

A dependência do álcool e de outras drogas pode provocar várias doenças, sendo mais incidentes as relacionadas ao aparelho digestivo. A incidência de efeitos psíquicos é também considerada, apresentando-se através de delírios e alucinações, manifestando perda do sentido de realidade, e sensação de perseguição (CAPISTRANO, 2013).

A cada ano são registradas cerca de 2,5 milhões de mortes relacionadas ao uso nocivo do álcool, ocupando o terceiro lugar no ranque de perdas de jovens no mundo. Para combater esse quadro, a OMS orienta que haja regulação do mercado de bebidas, especialmente para os jovens, regulando sua disponibilidade e acesso, implementando políticas apropriadas sobre bebidas e direção, aumentando $\mathrm{o}$ preço do produto $\mathrm{e}$ desenvolvendo de políticas públicas para combate ao uso (OMS, 2014).

Tavares, Béria e lima (2001) discorrem que os adolescentes consistem um dos maiores consumidores de álcool e drogas psicotrópicas, caracterizando, então, um grave problema de saúde pública, com variadas consequências pessoais para essa população.

De acordo com o II Levantamento Nacional de Álcool e Drogas (Laranjeira, 
2014), 52\% da população com idade maior que 18 anos faz uso de bebida alcoólica pelo menos uma vez ao ano, entre estes, $64 \%$ homens e $39 \%$ mulheres. O mesmo estudo revela que $11 \%$ da população fazuso diário, e $28 \%$, de três a quatro vezes por semana. Os dados apontam também que $3 \%$ da população com idade maior que 18 anos, faz uso nocivo de drogas psicotrópicas, e $9 \%$ é dependente dessas substâncias. Diante dos dados apresentados, observou-se a relação do uso abusivo de drogas psicotrópicas com patologias e transtornos mentais em geral, e também com cirrose hepática, pancreatite, câncer, além de estar associado à ocorrência de acidentes de trânsito e homicídios (LARANJEIRA, 2014).

Sob essa ótica, Guerra, Duarte e Oliveira (2010) ressaltam que, considerando a população jovem, os universitários têm recebido atenção especial, no que diz respeito a pesquisas. Fato que os autores atribuem às funções que os universitários possivelmente exercerão na sociedade e no desenvolvimento geral do país. Além disso, conhecer a prevalência de uso e a opinião dessa população sobre o uso de álcool e outras drogas, contribuirá para levantar potenciais informações e compreensão sobre seu comportamento.

Assim, este estudo objetivou avaliar o consumo do álcool e drogas entre os acadêmicos do curso de Psicologia de uma instituição privada de ensino superior na cidade de Montes Claros - MG, considerando a importância de se conhecer os principais fatores que levam os acadêmicos a iniciar o consumo dessas substâncias e as principais consequências acarretadas a sua saúde.

O estudo torna-se relevante na medida em que se propõe a identificar e reconhecer a realidade da referida população com relação ao consumo do álcool e outras drogas e, caso necessário, planejar ações voltadas à prevenção primária e secundária, considerando as necessidades dos acadêmicos, visando diminuir o consumo ou sanar possíveis fatores de risco.

A importância desse tema voltado para acadêmicos da área da saúde se intensifica quando se considera que, futuramente, esses sujeitos atuarão junto à população e deverão estimular a adesão de seus clientes a hábitos saudáveis, conforme pontua (NUNESet al.,2012).

\section{MATERIAIS E MÉTODOS}

O estudo foi aprovado pelo Comitê de Ética em Pesquisa (CEP) da Associação Educativa do Brasil - SOEBRAS sob o parecer $\mathrm{n}^{\mathrm{o}}$ 530.651/2014. Trata de uma pesquisa de caráter descritiva, com abordagem quantitativa e transversal,

A amostra foiconstituída por 295 participantes, ambos os sexos, selecionados aleatoriamente, na faixa etária de 20 a 45 anos,devidamente matriculados. Foram incluídos aqueles que aceitaram livremente 
participar da pesquisa, e excluídos os indivíduos que não aceitaram assinar o termo de consentimento,ou que não preencheram devidamente o questionário, e ainda aqueles que estavam ausentes no dia de aplicação do questionário, após três tentativas.

A coleta de dados foi realizada pelas pesquisadoras. Para tal, foram utilizados os instrumentos apresentados a seguir.

Questionário Sócio demográficoInstrumento autoaplicado, constituído por perguntas ordenadas, com respostas escritas, e sem a presença do pesquisador. $\mathrm{O}$ questionário é composto por questões discursivas e objetivas, visando obter as características gerais da amostra, como grau de instrução, estado civil, sexo, idade, renda familiar.

Teste para Identificação de Problemas Relacionados ao Uso de Álcool (AUDIT) Usado para avaliar o uso individual de álcool, problemas relacionados ao álcool e a dependência, sendo composto por dez questões de múltipla escolha. As respostas são pontuadas de zero a quatro e, ao final do teste, os pontos obtidos serão somados para concluir a pontuação final e a classificação de cada participante (MORETTI-PIRES; CORRADI-WEBSTER, 2011). A classificação é obtida relacionando as Zonas do AUDIT (Zonas I, II e III) e a pontuação alcançada pelo sujeito, conforme disposto pelo Ministério da Justiça (2014). Zona I (até7 pontos): uso de baixo risco ou abstêmia de álcool;Zona II (8 a 15 pontos): apresenta uso de risco, sem problema atual relacionado ao consumo. Zona III (16 a 19 pontos): padrão de uso nocivo com problemas decorrentes do uso de álcool; Zona IV (acima de 20 pontos): grande chance de diagnóstico de dependência de álcool.

O referido teste foi desenvolvido pela OMS, como um método simples de investigação do uso abusivo de álcool, sendo considerado eficiente para detectar também o uso nocivo do álcool, devido sua sensibilidade e especificidade, constituindo-seum instrumento de fácil aplicação, que permite resultado imediato e intervenções direcionadas a partir da zona de classificação identificada (GONÇALVES et al.,2015).

Inventário de Expectativas e Crenças Pessoais acerca do álcool (IECPA) Instrumentoconstituído por 61 itens com 5 alternativas em escala do tipo de Likert. Visa conhecer o que o participante pensa sobre o efeito do álcool, sendo as escalas de1 a 5, com as seguintes correspondências: 1 -"não concordo"; 2 -“concordo pouco"; 3 "concordo moderadamente"; 4 -“concordo muito" e 5 - “concordo muitíssimo". É adequado preferencialmente para autoaplicação com duração estimada em quinze minutos (AMARAL; SALDANHA, 2009).

O instrumento é aplicável em adolescentes e adultos; o escore final pode variar de 0 a 305 pontos. Os resultados mais 
elevados caracterizam participantes com expectativas positivas mais altas e, portanto, maior vulnerabilidade ao alcoolismo. São classificados comobaixa expectativa, aqueles que pontuam até 121, e alta expectativa, os que pontuam 122 ou mais (MARTINSet al.,2010).

Teste de Triagem do Envolvimento com Álcool, Cigarro e Outras Substâncias (ASSIST) - Instrumento em formato de questionário, produzido pela Organização Mundial de Saúde (OMS) para detectar o uso de álcool, tabaco e outras substâncias psicoativas. É utilizado por profissionais de saúde de diversas formações, pode-se considerar a abordagem simultânea de várias classes de substâncias, obtendo também a facilidade de interpretação (Henrique, 2004).

$\mathrm{O}$ teste em questão foi utilizado no intuito de complementar o, já padronizado, Teste para Identificação de Problemas Relacionados ao Uso de Álcool (AUDIT), também utilizado neste estudo. $\mathrm{O}$ teste ASSISTaborda problemas relacionados ao uso de drogas psicoativas, preocupação das pessoas próximas ao usuário, tentativas mal sucedidas de cessar ou reduzir o uso, prejuízos na execução de tarefas esperadas, sentimentos de compulsão e uso por via injetável (SOARES, et al., 2015).

Escala de Satisfação com o Suporte Social (ESSS) - Trata-se de um instrumento constituído por 15 afirmações relativas à satisfação com o suporte social,autoaplicado, apresentado em formato de resposta de Likert com 5 pontos. As alternativas propostas são: concordo totalmente, concordo na maior parte, não concordo nem discordo, discordo na maior parte e discordo totalmente. No estudo original, Ribeiro (1999) aponta que a escala foi aplicada em uma amostra de estudantes portugueses com a faixa etária de 15 a 30 anos, e na análise fatorial exploratória foram retidos 4 fatores que explicaram $63 \%$ da variância total. A consistência interna foi adequada Escala: $\alpha=0,85$; Fatores: $\alpha=0,64$ 0,83 (MARÔCOet al., 2014).

Este instrumento avalia um conjunto de medidas que expressam saúde, bem-estar, mal-estar ou que estão intimamente ligadas a essas variáveis. Para que o ESSS seja válido deriva-se da adaptação da escala portuguesa do Self perception profile for collegestudents, onde se propõe investigar a gravidade aos acontecimentos que o sujeito atribui a sua vida, analisando a intensidade do acontecimento que mais afetou a vida no último ano, além de um questionário sociodemográfico para caracterização do grupo amostral (SILVAet al.,2015).

Após a autorização para realização da pesquisa por parte da direção da instituição, foi feito uma reunião com os acadêmicos, a fim de esclarecer os objetivos, justificativa e metodologia do estudo, além de sanar possíveis duvidas. Os questionários foram aplicados pelas próprias pesquisadoras nos meses de março e abril 2016, sempre em 
horários extra-aulas e em sala reservada para esse fim.

Os dados analisados de forma descritiva, com valores em porcentagem, o procedimento estatístico foiefetuado no SPSS (StatisticalPachage for Social Sciences), versão 22.0 para Windows.

\section{RESULTADOS E DISCUSSÃO}

A amostra foi constituída por 295 acadêmicos na faixa etária de 20 e 45 anos, distribuídos entre o $1^{\circ}$ e $11^{\circ}$ períodos do curso de Psicologia, no primeiro semestre de 2016. Os resultados apresentam maior participação do $4^{\circ}$ período $(17,56 \%)$, seguido do $8^{\circ}$ período $(16,89 \%)$, e menor participação do $9^{\circ}$ período $(0,67 \%)$. Os resultados obtidos a partir da aplicação dos questionários estão apresentados nas tabelas abaixo.

Tabela 1 - Caracterização do Grupo Amostral (n= 295)

\begin{tabular}{llll}
\hline Variável & & N & \% \\
\hline Sexo & Masculino & 51 & 17,23 \\
& Feminino & 245 & 82,77 \\
Etnia & Asiático & 2 & 0,68 \\
& Branco & 82 & 27,8 \\
& Índio & 5 & 1,7 \\
& Negro & 37 & 12,55 \\
& Pardo & 169 & 57,29 \\
& Solteiro & 207 & 70,17 \\
\hline
\end{tabular}

\begin{tabular}{llll}
\hline & Casado & 40 & 13,56 \\
& Separado & 10 & 3,39 \\
& Viúvo & 0 & 0 \\
& Namoro & 38 & 12,89 \\
Período & $1^{\text {o }}$ & 35 & 11,82 \\
& $2^{\text {o }}$ & 26 & 8,7 \\
& $3^{\text {o }}$ & 35 & 11,82 \\
& $4^{\text {o }}$ & 52 & 17,56 \\
$5^{\circ}$ & 27 & 9,12 \\
$6^{\circ}$ & 12 & 4,05 \\
$7^{\circ}$ & 16 & 5,4 \\
$8^{\text {o }}$ & 50 & 16,89 \\
$9^{\circ}$ & 2 & 0,67 \\
$10^{\circ}$ & 20 & 6,75 \\
$11^{\circ}$ & 21 & 7,09 \\
\hline
\end{tabular}

Fonte: Próprios autores

Os dados apresentados na tabela 1 indicam o grupo amostral de 295 acadêmicos, no qual, verifica-se que $82,77 \%$ são do sexo feminino e $17,23 \%$ do sexo masculino, salientandouma discrepância quanto aos sexos, com maior prevalência do sexo feminino.Resultados semelhantes foram apresentados em outros trabalhos sobre o consumo de álcool (SILVAet al., 2014;SILVAet al., 2015).

O predomínio de sujeitos do sexo feminino é justificado por Costa, Durães e Abreu (2010) que ressaltam que, na atualidade, as mulheres constituem maioria no ensino superior, o que representa um marco nas mudanças sociais, considerando que a 
universidade já foi considerada espaço masculino.

Com relação à etniaobserva-se uma mesclacom maior evidencia para pardos (57,29\%), seguido de brancos $(27,8 \%)$ enegros $(12,55 \%)$,e uma pequena amostra declarada índio e asiático. Esse resultado assemelha-se ao apresentado em outros estudos (SILVA et al., 2015;SOARESet al., 2015), e são justificados pelamiscigenação presente no Brasil (SILVAet al., 2015).

No que se refere ao estado civil, maioria dos participantes $(70,17 \%)$ se declarou solteiros. Esse resultado é consoante com os estudos deSilva (2015) e Soares et al. (2015), os quais apresentaram, respectivamente, $64,4 \%$ e $55,07 \%$ de estudantes declarados solteiros.

Estudos feitos com a população brasileira apontam que normalmente o consumo moderado de álcool é uma dose por dia para as mulheres e duas doses por dia para os homens. Alguns fatores são apontados como facilitadores para o consumo excessivo de álcool e tabaco no Brasil, entre eles, o nível de escolaridade mais elevado, melhor situação socioeconômica, e inserção no mercado de trabalho. As regiões sul e sudeste do país apresentam crescimento do consumo excessivo dessas substâncias entre o sexo masculino, classificados na etnia negra $\mathrm{e}$ parda, solteiros, sem religião, de nível social baixo. Os estudos mostram também que na região nordeste não se verificou associação entre o padrão de consumo e cor de pele.Em pesquisa feita na região norte de Minas Gerais com acadêmicos de Psicologia, registrou-se baixo risco de consumo de álcool e outras drogas, observando prevalência $(57,29 \%)$ em pessoas que se consideram pardas (CARDOSO; MELO; CESAR,2015).

A classificação registrada pelo presente estudo segue apresentada na Tabela 2.

Tabela 2 - Resultados a partir dos dados dos questionários

\begin{tabular}{lllll}
\hline QUESTIONÁRIO & NÍVEL & $\mathbf{N}$ & $\%$ & CLASSIFIC \\
& & & & ACÃo
\end{tabular}

\begin{tabular}{|c|c|c|c|c|}
\hline AUDIT & 1 & 247 & 83,1 & Baixo \\
\hline & & & 8 & Risco \\
\hline & 2 & 42 & 14,1 & Médio \\
\hline & & & 4 & Risco \\
\hline & 3 & 6 & 2,02 & Alto Risco \\
\hline & 4 & 2 & 0,67 & Dependênc \\
\hline & & & & ia Álcool \\
\hline ASSIST & 1 & 241 & 81,1 & Uso \\
\hline & & & 4 & Ocasional \\
\hline & 2 & 56 & 18,8 & Uso \\
\hline & & & 6 & Abusivo \\
\hline IECPA & 1 & 244 & 82,1 & Baixa \\
\hline & & & 5 & Expectativa \\
\hline & & & & - Baixa \\
\hline & & & & vulnerabili \\
\hline
\end{tabular}




\begin{tabular}{|c|c|c|c|c|}
\hline & & & & dade \\
\hline & 2 & 53 & 17,8 & Alta \\
\hline & & & 5 & Expectativa \\
\hline & & & & - Alta \\
\hline & & & & vulnerabili \\
\hline & & & & dade \\
\hline \multirow[t]{9}{*}{ ESSS } & 1 & 1 & 0,34 & Baixo \\
\hline & & & & Suporte \\
\hline & & & & Social \\
\hline & 2 & 118 & 39,7 & Médio \\
\hline & & & 3 & suporte \\
\hline & & & & social \\
\hline & 3 & 178 & 59,9 & Alto \\
\hline & & & 3 & Suporte \\
\hline & & & & Social \\
\hline
\end{tabular}

Fonte: Próprios autores

A experiência universitária permite ao jovem fazer parte de um enorme grupo de pares, sem supervisão familiar, tornando-os mais vulneráveis a fazer o uso de álcool e de drogas lícitas e ilícitas. O álcool consiste a substância mais consumida pelos jovens, seguida pelo tabaco, maconha e estimulantes. O uso dessa substância pode levar o jovem a cometer algumas atitudes inadequadas, como beber e dirigir, atividade sexual desprotegida, violência, causando risco para sua saúde e aumentando o problema de saúde pública(PEDROSAet al., 2011).

Embora estudos apontem que o consumo de álcool e de outras drogas cresce entre universitários e se constituam causa de sérios problemas de saúde pública, os resultados apresentados neste estudo (Tabela 2) com relação à classificação de risco (questionário AUDIT)apontam que 83,18\% da amostra apresentabaixo risco de problemas relacionados ao álcool e dependência, sendo classificados como nível 14,14\%apresenta médio risco, classificados como nível (2), 2,02\% apresenta alto risco classificados como nível (3) e por fim classificados como nível (4) foram apenas $0,67 \%$ dependentes de álcool.

Esse resultado é consoante com estudo voltado para acadêmicos do curso de Tecnólogo em Gastronomia, na mesma instituição de ensino, o qual apresenta que a maioria dos acadêmicos $(78,05 \%)$ foi classificada com baixo risco, nível (1), 14,63\% apresentou médio risco enível (2), $4,88 \%$ classificou-se como alto risco, nível (3), e 2,44\% com dependência de álcool, nível (4) (FIGUEIREDOetal.,2015).

O número de dependentes químicos no Brasil cresce a cada dia, e há uma escassez de tratamento público eficaz, pois os Centros de Atenção Psicossociais (CAPS), serviços governamentais implantados há pouco tempo, encontram dificuldades mediante a falta de experiência da gestão nos serviços, bem como, com a falta de profissionais qualificados e de serviços especializados, tornando-se um serviço de baixa resolutividade e baixa eficácia. É necessário implementarserviços com diferentes níveis de 
complexidade para melhor atender o paciente nas diversas fases do processo de recuperação, pois cada paciente possui sua subjetividade, e seu projeto terapêutico, observando a necessidade de um serviço específico para cada paciente, tais como, serviços para desintoxicação, para intervenção na crise, ambulatórios, tratamento das comorbidades psiquiátricas relacionadas ao uso de substâncias, e para apoio social (Silva, 2013).

Os resultados encontrados no ASSIST evidenciam que $81,14 \% \quad(n=241) \quad$ dos avaliados, relataram fazer uso ocasional de bebidas alcoólicas, e 18,86\% fazem uso abusivo. Resultado semelhante foi encontrado em estudo voltado para acadêmicos do curso de Direito de uma instituição privada, no qual, 91,09\%dos acadêmicos registraram fazer uso de bebidas alcoólicas ocasionalmente, enquanto $8,91 \%$ relataram uso abusivo (SILVA etal.,2015).No que se refere ao uso abusivo, o presente estudo apresenta um resultado significativo $(18,86 \%)$. O registro de dependentes químicos na amostra é baixo, o que significa que os sujeitos desta pesquisa não estarão frequentando o CAPS, o que é importante, considerando que esse serviço não possui estrutura adequada receber grande número de usuários e oferecer o tratamento.

Os resultados do teste IECPA registram que maioria dos acadêmicos $(82,15 \%)$ apresentarabaixa vulnerabilidade aos efeitos do álcool, e 17,85\% altavulnerabilidade. Esse resultado encontra semelhança em estudo apresentado por Silva et al. (2014) com estudantes do curso de Medicina Veterinária, o qual avaliou quea maior parte da amostraregistroualta vulnerabilidade aos efeitos do álcool.

Formigaet al.(2013) ressaltamque jovens e universitários que apresentam uma autoestima positiva tendem a apresentar baixo risco ao uso excessivo e dependência de drogas lícitas e ilícitas, e pessoas com baixa autoestima tendem a se refugiar em drogas, para possivelmente aliviar o sofrimento.

De acordo com a amostra obtida pelo ESSS, $59,93 \%$ dos universitários evidenciaram alto suporte social, sendoqualificados como nível 3. Este resultado diverge de estudo voltado para acadêmicos do curso de Administração em instituição de ensino superior privada em Minas Gerais, no qual, $61,61 \%$ dos voluntários demonstraram médio suporte social, classificados como nível 2.

Os instrumentos que avaliam o suporte social no Brasil são considerados escassos, e os existentes são adaptados para nossa realidade. Observa-se a necessidade de implementar instrumentos específicos para atender a população. $\mathrm{O}$ apoio social é importante na vida do sujeito, tanto para aquele que o oferece, quanto para quem recebe, sendo mais impactante para o indivíduo que recebe, podendo causar desfechos positivos emsua saúde física e 
mental, bemestar emocional. Para os usuários de drogas, lícitas ou ilícitas, esse suporte torna-se ainda mais importante, diante de relatos de que, muitas vezes, o uso dessas substâncias inicia-se mediante problemas de relacionamento com pessoas próximas e importantes na sua vida social do sujeito, o que pode influenciar na perda de a autoestima, senso de controle e o domínio sobre a própria vida. Assim, o apoio social se constitui como crucial na vida de qualquer sujeito, em conjunto com as características psicologias, emocionais, físicas, e nas estratégias de enfrentamento e a avaliação subjetiva da situação (GONÇALVESet al.,2011).

\section{CONCLUSÃO}

\section{REFERÊNCIAS}

AMARAL, A. C., SALDANHA, A. A. Parâmetros psicométricos do Inventário de Expectativas e Crenças Pessoais acerca do Álcool para adolescentes. Psico- USF,v.14, n.2, p.167-176, 2009.

CARDOSO, L. G. V., MELO, A. P., CESAR, C.C.Prevalência do consumo moderado e excessivo de álcool e fatores associados entre residentes de Comunidades Quilombolas de Vitória da Conquista, Bahia, Brasil.

Rev. Ciências\& Saúde Coletiva,v.20, n.3, p.809-820, 2015.

CAPISTRANO, F. C., KALINKE, L. P., FERREIRA, A. C., MAFTUM, M. A., SILVA, T. L. Perfil sociodemográfico e clínico de dependentes químicos em tratamento: análise de prontuários. Escola Anna Nery,v.17, n.2, p.234-241, 2013.
Os resultados alcançados neste estudo evidenciaram o predomínio de baixo risco de problemas relacionados ao uso de álcool e outras drogas, baixa vulnerabilidade aos efeitos do álcool e alto suporte social,o que sugere que o consumo dessas substâncias nessa amostra é baixo, bem como há uma baixa estimativa de desenvolvimento de alcoolismo. Entretanto, 18,86\%, da amostra registrou uso abusivo de álcool e 0,67\% registrou dependência. Nesse contexto, é importante que a instituição invista em campanhas voltadas à conscientização dos problemas advindos do uso e abuso dessas substâncias, bem como trabalhar com a prevenção. É importante que novos estudos sejam levantados, visando o acompanhamento da trajetória desses estudantes.

CEBRID. (2013). Centro Brasileiro de Informações sobre Drogas Psicotrópicas. Livreto informativo sobre drogas psicotrópicas. 5ed. São Paulo: CEBRID, 2013.

COSTA, S. M., DURÃES, S. J. A., ABREU, M. H. N. G. Feminização do curso de odontologia da Universidade Estadual de Montes Claros. Ciênciae Saúde Coletiva, v. 15(supl. 1), p.1865-1873, 2010.

FIGUEIREDO, O. A. C., SOUZA, J. D., MARIE JONES, K., SOARES, P. K., FINELLI, L. A., SOARES, W. D.(2015).Utilização do Álcool como MediadorSocial em Acadêmicos doCurso de Tecnólogo emGastronomia.Revista Humanidades, v.4, n.2, p.42 -51, 2015.

FORMIGA, N. S., PICANÇO, E., SOUZA, R. C., SANTOS, J. D.Identificação do problema com o consumo alcoólico em 
pessoas vulneráveis e não vulneráveis e sua relação com autoestima. Psicologia PT - O Portal dos Psicólogos,p.01-18, 2013.

GONÇALVES, T. R., PAWLOWSKI, J., BANDEIRA, D. R., PICCININI, C. A. Avaliação de apoio social em estudos brasileiros: aspectos conceituais e instrumentos.Rev. Ciência e Saúde coletiva, v.16, n.3, p.1755-1769, 2011.

GONÇALVES, A., OLIVEIRA, A., GANDRA, H., ASSUNÇÃO, P., OLIVEIRA, T., SILVA, T.Avaliação do padrão de uso do álcool entre morador de uma região socialmente vulnerável.Revista Portuguesa de Enfermagem de Saúde Mental, (Esp). n.2, p.95-100, 2015.

GUERRA, A. A., DUARTE, P. C., OLIVEIRA, L. G. (ORGS). I Levantamento nacional sobre o uso de álcool, tabaco e outras drogas entre universitários das 27 capitais brasileiras. Brasília, DF: SENAD. 2010.

HENRIQUE. I. F.Validação da versão brasileira do teste de triagem do envolvimento Com álcool, cigarro e outras substâncias (ASSIST). Revista Associação Médica Brasileira, v.50, n.2, p.199-206, 2004.

LARANJEIRA, R., PINSKY, I., ZALESKI, M., CAETANO, R.I Levantamento Nacional sobre os padrões de consumo de álcool na população brasileira. Brasília: SENAD, 2007.

LARANJEIRA, R. (Org.). II Levantamento Nacional de Álcool e Drogas (LENAD). São Paulo: INPAD/UNIFESP, 2014

LARANJEIRA, R. (Superv.).(2012). Levantamento Nacional de álcool e drogas.Instituto Nacional de Ciência e Tecnologia para Políticas Públicas do Álcool e Outras Drogas.São Paulo: INPAD/UNIFESP, 2012.

MALTA, D. C., MASCARENHAS, M. D., PORTO, D. L., DUARTE, E. A., SARDINHA, L. M., BARRETO, S. M.,
NETO, O. L. Prevalência do consumo de álcool e drogas entre adolescentes: análise dos dados da Pesquisa Nacional de Saúde Escolar. Revista Brasileira de Epidemiologia, v.14, n.1, p.136-146, 2011.

MANGUEIRA, S. O. Promoção da saúde e políticas públicas do álcool no Brasil: revisão integrativa da literatura. Psicologia e Sociedade,v.27, n.1, p,157-168, 2014.

MARÔCO. J. P., CAMPOS, J. A., VINAGRE, M. G., PAIS-RIBEIRO, J. L. Adaptação transcultural Brasil-Portugal da Escala de Satisfação com o Suporte Social para estudantes do ensino superior.Psicologia: Reflexão e Crítica,v. 27, n.2, p. 247-256, 2014.

MARTINS, R . A., PARREIRA, G. V., CRUZ, L. A., SILVA, I. A.Expectativas Sobre os Efeitos do Uso deÁlcool e Padrão de Beber em Alunos de Ensino Médio.Revista Eletrônica Saúde Mental Álcool e Drogas, 6(1),2-13, 2010.

\section{MARTINS, L. Curso de Extensão}

Prevenção em Pauta: Conceitos Básicos Sobre Drogas e seus Padrões de Consumo. Belo Horizonte: [s.n], Secretaria de Estado de Saúde de Minas Gerais, 2011.

MORETTI-PIRES, R., CORRADIWEBSTER, C.Adaptação e validação do Alcohol Use DisorderIdentification Test (AUDIT) para população ribeirinha do interior da Amazônia, Brasil. Caderno de Saúde Pública, v.27, n.3, p.497-509, 2011.

NICASTRI, S. Drogas: classificação e efeitos no organismo. In Brasil. Prevenção ao uso indevido de drogas: Curso de Capacitação para Conselheiros Municipais. 4ed. Brasília: SENAD, 2011.

NUNES, J. M., CAMPOLINA, L. R.,VIEIRA, M. A., CALDEIRA, A. P.Consumo de bebidas alcoólicas e prática do bingedrinking entre acadêmicos da área da saúde. Revista PsiquiatriaClinica,v.39, n.3, p.94-102, 2012. 
PEDROSA, A. A., CAMACHO, L. A.,

PASSOS, S. R., OLIVEIRA, R. V. Consumo

de Álcool entre estudantes

Universitários.Revista Cadernos de Saúde

Pública, v.27, n.8, p.1611-1621, 2011.

SILVA, C. J.Dependência Química no Brasil e o papel das Organizações Sociais na gestão dos serviços de tratamento: estudo do Caso UNAD - Unidade de Atendimento ao Dependente Químico.Instituto de Ensino e Pesquisa, p.01-90, 2013.

SILVA, D. M., PIMENTA, L. M., MARIE JONES, K., VIANNA, R. S., FINELLI, L. A., SOARES, W. D.(2014). Consumo de álcool entre acadêmicos do Curso de Medicina Veterinária. Revista Humanidades, v.3, n.1, p.19 -27, 2014.

SILVA, E. M., SOUZA, M. C., MARIE JONES, K., SOARES, W. D. Consumo de álcool entre os acadêmicos do curso de direito.Revista Eletrônica Gestão

\&Saúde,v.6, n.3, p.2723-2738, 2015.

SILVA, J. N., RODRIGUES, M. G., MARIE JONES, K., FINELLI, L. A., SOARES, W. D.(2015). Consumo de álcool entre universitários. Revista Brasileira de Pesquisa em Ciência da Saúde,v.2, n.2, p.35-40, 2015

SOARES, W. D., SILVA, H. D., PEREIRA, M. S., MARIE JONES, K., FINELLI, L. A., ALMEIDA, P. N., SOARES, P. K.Consumo de álcool entre acadêmicos de enfermagem.Revista Norte Mineira de Enfermagem, v.4, n.1, p.29-41, 2015.

TAVARES, B. F., BÉRIA, J. U., LIMA, M. S. Prevalência do uso de drogas e desempenho escolar entre adolescentes.

Revista de Saúde Pública, v.35, n.2, p.150-

158. 2001. 